\title{
Comparative morphology and phenetics of Nymphoides species in Kerala
}

\author{
Pavisha P*, Pradeep N S \& Madhusoodanan P V \\ KSCSTE-Malabar Botanical Garden and Institute for Plant Sciences (MBGIPS), Kozhikode 673 014, Kerala, India \\ *Email: pavishapongott@gmail.com
}

\section{ARTICLE HISTORY}

Received: 23 January 2020

Accepted: 31 March 2020

Published: 02 May 2020

\section{KEYWORDS}

Nymphoides

Menyanthaceae

phenetics

dendrogram
ABSTRACT

Aquatic plants play an important role in maintaining the ecosystem balance. Nymphoides Seguier (Menyanthaceae) is an emergent, rhizomatous or stoloniferous genus, commonly known as "floating hearts", identified by its rounded or cordate, floating leaves, petiole like branches and unique floral characters. The present study deals with the comparative morphology and the phenetics of Nymphoides spp. in Kerala. These species are classified into two groups with the help of the dendrogram, which showed $66 \%$ dissimilarities among the two groups. $N$. parvifolia and $N$. balakrishnanii are closely related species in one group and N. krishnakesara and N. macrosperma are closely related species in other group.

\section{Introduction}

Menyanthaceae Dumort. are the most diverse and widespread family of aquatic and wetland plants under the order Asteralses. The family has five genera with 60-70 species distributed all over the world. The five genera are Menyanthes L., Liparophyllum Hook. f., Nephrophyllidium Gilg., Villarsia Vent. and Nymphoides Seguier. Of these five genera, Nymphoides are cosmopolitan in distribution. Menyanthes and Nephrophyllidium are restricted to Northern Hemisphere, Liparophyllum and Villarsia are found in Southern Hemisphere (11). The genus Nymphoides can be identified by its rounded or cordate, floating leaves, petiole like branches and its floral characters. Nymphoides species are both dioecious and monoecious.

The genus Nymphoides has 40-50 species in world, which are found in tropical regions of Africa, Australia, the Americas, India, and southeastern Asia $(1,5-8,11)$. Eight species are found in India, seven of which are growing in Kerala (8). Recently two new species and one variety are reported $(2,3)$. The present study deals with the comparative morphology and phenetics of seven Nymphoides species in Kerala.

\section{Materials and Methods}

Seven Nymphoides spp. were collected from different parts of Kerala and conserved in the aquatic plant conservatory (Aquagene) of the Malabar Botanical Garden and Institute for Plant Sciences. The morphological and phylogenetic characters of the specimens were compared on the basis of visible characters. The phylogeny of seven species of Nymphoides in Kerala was done (Fig. 1 \& Fig. 2).

The data for the analysis were collected from fresh material collected from different localities and also from herbarium specimens deposited in the Central National Herbarium (CAL), Botanical Survey of India, Southern Regional Centre (MH), Calicut University Herbarium (CALI) and Malabar Botanical Garden Herbarium (MBGH). In the present study, twenty two multi-state qualitative morphological characters of the Nymphoides spp. were considered (Table 1) and gave code numbers to the seven species (Table 2). The characters (with abbreviated codes) used in the cluster analysis with their character states are given in Table 1 . The characters with two states were coded as 1 and 2 and also given continuous numbers when the character states are more than two. The results of multiple

\footnotetext{
(c) Pavisha et al. (2020). This is an open-access article distributed under the terms of the Creative Commons Attribution License, which permits unrestricted use,
} distribution, and reproduction in any medium, provided the original author and source are credited (https://creativecommons.org/licenses/by/4.0/).

To cite this article: Pavisha P, Pradeep NS, Madhusoodanan PV. Comparative morphology and phenetics of Nymphoides species in Kerala. Plant Science Today. 2020;7(2):233-239. https://doi.org/10.14719/pst.2020.7.2.735 

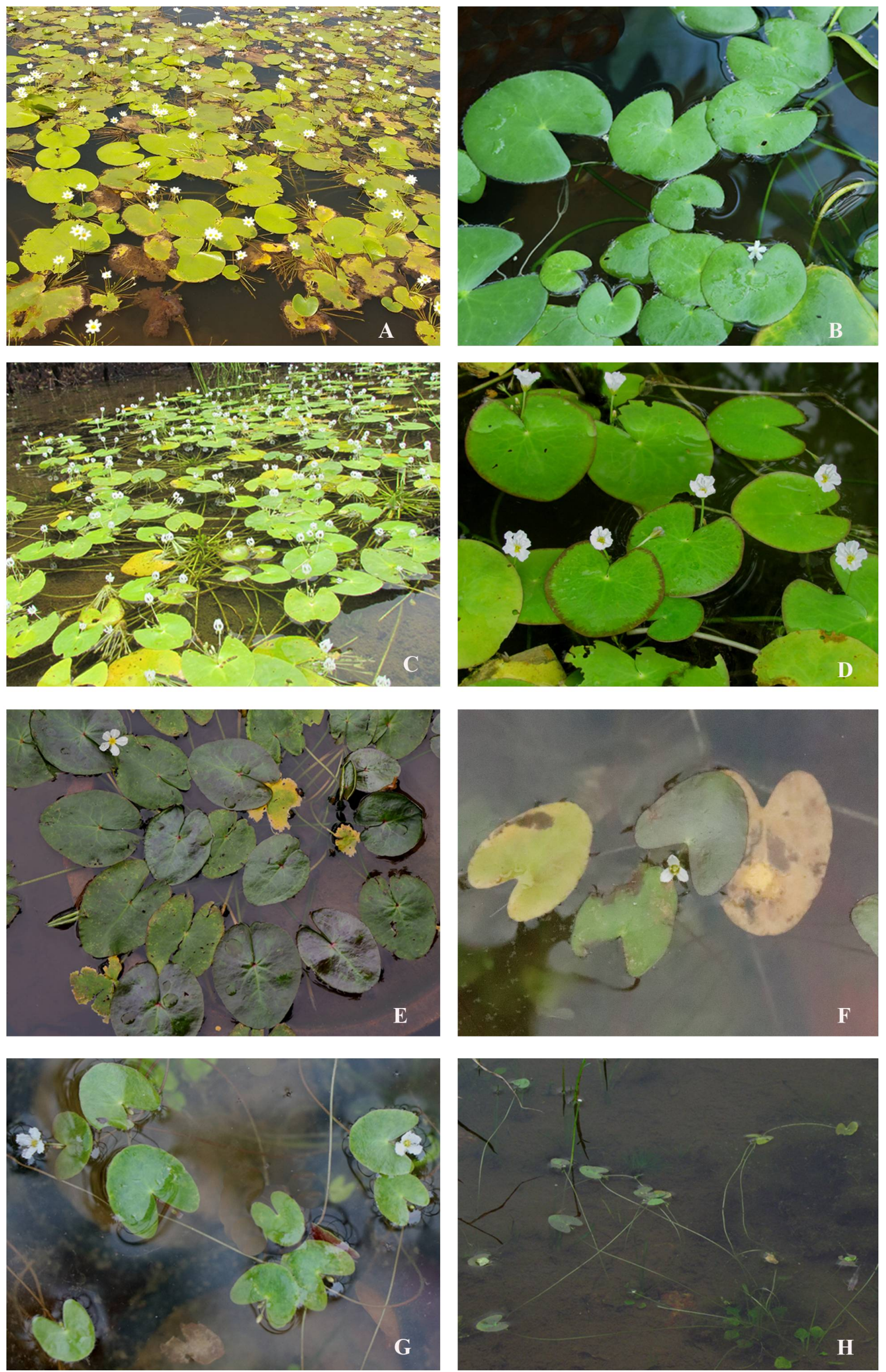

Fig.1. Nymphoides species in Kerala. A. $N$. indica (L) Kuntze, B. N. macrospema R.V.Nair, C. . krishnakesara K.T. Joseph \& Sivar., D \& E. N. hydrophylla (Lour.) Kuntze, F. N. parvifolia Kuntze, G. N. balakrishnanii Biju, Josekutty, Haneef \& Augustine., H. N. palyii Biju, Josekutty, Haneef \& Augustine

range tests of characters of the OTUs are given in the Table 3. For cluster analysis, all the twenty two characters were tabulated against the seven OTUs using the numerical codes given for character 

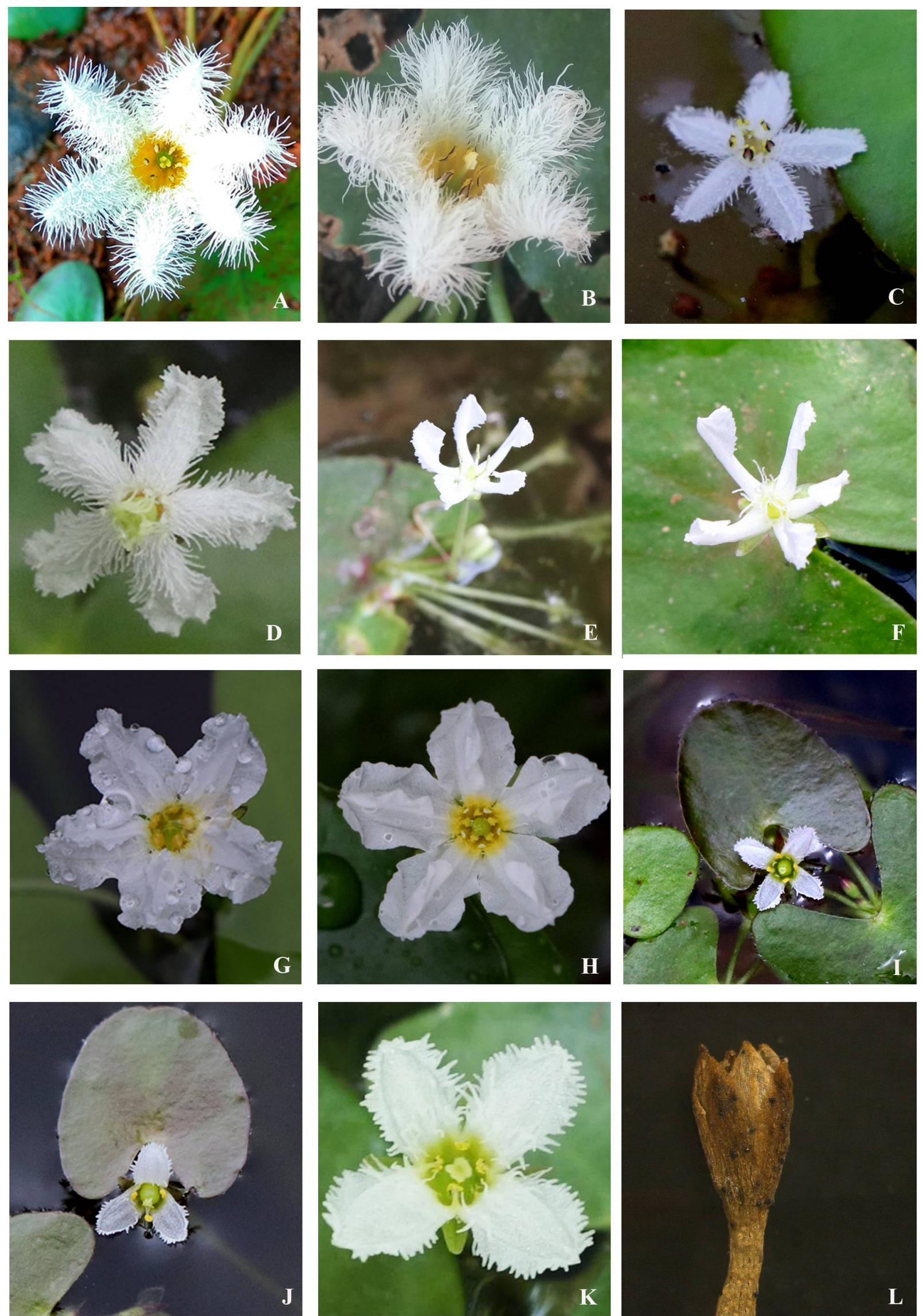

Fig. 2. Flowers of Nymphoides species in Kerala. A \& B. Short styled and Long styled flowers of $N$. indica, C \& D. Male and Female flowers of $N$. macrosperma, E \& F. Male and Female flowers of $N$. krishnakesara, G \& H. Bisexual \& Female flowers of N. hydrophylla, I \& J. Flowers of N. parvifolia, K. N. balakrishnanii flower, L. Flower of N. palyii (Herbarium)

states and abbreviated codes of characters (Table 4). These data were used to generate dendrogram using the statistical package STATISTICA version
7.0, adopting Unpaired Group Method with Arithmetic mean as algorithm (9) and percent disagreement (4) as the statistical test. 
Table 1. Characters used in the cluster analysis

\begin{tabular}{llll}
\hline $\begin{array}{l}\text { Sl. } \\
\text { No. }\end{array}$ & Code & Characters & Characters states and their code numbers \\
\hline 1. & Hab & Habitat & Clay soil (1) or lateritic soil (2), clay soil and lateritic soil (3) \\
\hline 2. & Plt & Plant & Bisexual (1) or Unisexual (2), Bisexual and female (3) \\
\hline 3. & Rhi & Rhizome & Stoloniferous (1) or non stoloniferous (2) \\
\hline 4. & Sht & Shoot & Dimorphic (1) or monomorphic (2) \\
\hline 5. & Lvs & Leaves & Dimorphic (1) or Monomorphic (2) \\
\hline 6. & Flr & Flower & Bisexual (1), unisexual (2), bisexual or unisexual (3) \\
\hline 7. & ClxL & Calyx lobe & Oblong-acute (1), oblong-obtuse (2), linear- lanceolate (3), elliptic-lanceolate (4) \\
\hline 8. & Co & Corolla & White with yellow throat (1) or white (2) \\
\hline 9. & PetL & Petal lobe & Fimbriately toothed (1), shallowly fimbriate or undulate (2) \\
\hline 10. & PetLS & Shape of petal lobe & Obtuse or retuse (1), ovate to lancelate \\
\hline 11. & HrPL & Presence of hairs on petal lobe & (2), oblong-obtuse (3), oblong or elliptic (4) \\
\hline 12. & Stm & Stamen & Present (1) or absent (2) \\
\hline 13. & ClrA & Colour of Anther & Dimorphic (1) or monomorphic (2) \\
\hline 14. & NoSt & No. of stamens & Yellow (1), pale purple (2), blue (3), black with yellow (4), Cream (5) \\
\hline 15. & ISG & Inter staminal gland & Three or four (1), four (2), five (3), four to eight (4) \\
\hline 16. & ArC & Stigmatic hair & Present (1) or absent (2) \\
\hline 17. & Stl & Style & Present (1) or absent (2) \\
\hline 18. & HgG & Hypogynous gland & Heterostylous (1) or homostylous (2) \\
\hline 19. & Fr & Fruit & Hairy (1), hairless (2) \\
\hline 20. & Se & Seed & Ellipsoid (1), sub globose (2), obovoid (3), oblong (4), or oblong to obovoid (5) \\
\hline 21. & SeS & Shape of seed & Tuberculate (1) or smooth (2) \\
\hline 22. & PoS & Pollen shape & Discoid (1), obovate or elliptical (2), Obovoid (3) \\
\hline & & Prolate-spheroidal (1), prolate-spheroidal to subprolate (2) or oblate-spheroidal (3) \\
\hline
\end{tabular}

\section{Phenetics}

Phenetics (also known as taximetrics) is a method to classify organisms based on the overall morphological similarity notwithstanding the evolutionary relationships/ characters. In the present study the results of multiple range tests of characters of the OTUs are given in the Supplementary Table 1. For cluster analysis, all the twenty two characters were tabulated against the seven OTUs using the numerical codes given for character states and abbreviated codes of twenty two characters.

Table 2. Nymphoides spp. (OTUs) selected for the study

\begin{tabular}{cl}
\hline $\begin{array}{c}\text { Code } \\
\text { No. }\end{array}$ & Name of the species (OTUs) \\
\hline 1. & Nymphoides indica (L.) Kuntze \\
\hline 2. & Nymphoides macrosperma R.V.Nair \\
\hline 3. & Nymphoides krishnakesara K.T. Joseph \& Sivar. \\
\hline 4. & Nymphoides hydrophylla (Lour.) Kuntze \\
\hline 5. & Nymphoides parvifolia Kuntze \\
\hline 6. & $\begin{array}{l}\text { Nymphoides balakrishnanii Biju, Josekutty, Haneef \& } \\
\text { Augustine }\end{array}$ \\
\hline 7. & Nymphoides palyii Biju, , Josekutty, Haneef \& Augustine \\
\hline
\end{tabular}

\section{Results and Discussion}

The genus Nymphoides in Kerala state is represented by nine species and one variety, namely, Nymphoides aurantiacum, N. balakrishnanii, N. hydrophylla, N. indica, N. krishnakesara, N. krishnakesara var. bispinosa, $N$. macrosperma, $N$. parvifolia, $N$. palyii and $N$. sivarajanii. Eventhough after extensive explorations in the previous known localities, we could not relocate $N$. aurantiacum, $N$. krishnakesara var. bispinosa and $N$. sivarajanii and hence, they were not included in the present study. The worst flood occurred in Kerala state in 2018 may also adversely affected the fragmented population of these highly vulnerable species.
Key to the species of Nymphoides in Kerala

1a Flowers corolla upto $40 \mathrm{~mm}$ in diam., distylous; corolla upper side totally covered with dense hairs.

1b Flowers corolla upto $22 \mathrm{~mm}$ in diam., small, homostylous; corolla partially or not covered with hairs

2a. Plants dioecious; stigma bilobed with a whorl of radiating glandular hairs

$N$. indica

2b. Plants monoecious or gynodioecious; stigma bilobed without a whorl of radiating glandular hairs

3a. Shoot dimorphic, many jointed each joint bearing single leaf; leaves monomorphic; corolla lobes without median wings; hypogynous glands hairy

3b. Shoot monomorphic, single leaf arising from the axils of rhizome; leaves dimorphic; corolla lobes with median wings; hypogynous glands not hair

4a. Flowers unisexual (female) or bisexual; corolla lobes not fimbriate, undulate margine with upper median wing; seeds $2 \mathrm{~mm}$ across.

4b. Flowers bisexual; corolla lobes fimbriate without median wing; Seeds small, less than $2 \mathrm{~mm}$ across

5a. Petals 4 in number; floating leaves obovate - orbicular; calyx exceeds the fruit

5b. Petals 3 or 4 in number; floating leaves ovate - orbicular; calyx not exceeds the fruit

6a. Floating leaves pale green brownish green with pinkish tinge; hypogynous gland present; seed surface tuberculated

6b. Floating leaves dark green; hypogynous gland not distinct; seed surface smooth

N. macrosperma

N. krishnakesara

N. hydrophylla

N. balakrishnanii

N. parvifolia

N. palyii 
The result of comparative morphology of the seven Nymphoides spp. is given in Supplementary Table 1. The dendrogram of seven Nymphoides spp. in Kerala was obtained through the cluster analysis, using character states of the OTUs and resulted the percent disagreement between OTUs (Table 5 \& Fig. 3 . The results of dendrogram clearly revealed that the seven species come into two distinct groups Group I and Group II.

\section{Group 1: 1, 5, 6 \& 7}

Group II: 2, 3 \& 4

Group I and Group II were clustered together and show 66\% dissimilarities. Group I is classified into Group IA and Group IB. In Group I Nymphoides indica is clustered distantly from other species and shows 55\% dissimilarities from other species viz.,
Nymphoides parvifolia, $N$. balakrishnanii and $N$. palyii. Group IB is again classified into Group $\mathrm{IB}_{1}$ and Group $\mathrm{IB}_{2}$. N. palyii is come under Group $\mathrm{IB}_{2}$ and are separated from $N$. balakrishnanii and $N$. parvifolia (Group $\mathrm{IB}_{1}$ ). N. palyii shows $34 \%$ dissimilarities with other two species. These two species come in Goup $\mathrm{IB}_{1}$ are closely related, which shows highest affinities (68\% similarities).

Group II consists of two clusters Group IIA and Group IIB and represents three taxa viz., $N$. hydrophylla, $N$. krishnakesara, N. macrosperma. Group IIB is separated from Group IIA which consists of two taxa viz., $N$. krishnakesara and $N$. macrosperma. Group IIB is $60 \%$ dissimilar with Group IIA. In this study N. krishnakesara is clustered together with $N$. macrospermum instead of $N$. hydrophylla. N. krishnakesara shows more similarity (50\%) with $N$. macrosperma than $N$. hydrophylla. On

Table 3. Results of multiple range test of characters

\begin{tabular}{|c|c|c|c|c|c|c|}
\hline \multirow{2}{*}{$\begin{array}{l}\text { Code for } \\
\text { Characters }\end{array}$} & \multirow[t]{2}{*}{ Characters } & \multicolumn{5}{|c|}{$\begin{array}{l}\text { Grouping of Taxa (represented by code numbers) } \\
\text { according to their character states }\end{array}$} \\
\hline & & 1 & 2 & 3 & 4 & 5 \\
\hline $\mathrm{Hab}$ & Habitat & $\begin{array}{l}\text { Clay soil } \\
2,4\end{array}$ & $\begin{array}{l}\text { Laterite soil } \\
3,5,6,7\end{array}$ & $\begin{array}{l}\text { Caly soil and laterite } \\
\text { soil } \\
1\end{array}$ & & \\
\hline Plt & Plant & $\begin{array}{l}\text { Bisexual } \\
1,5,6,7\end{array}$ & $\begin{array}{l}\text { Unisexual } \\
2,3\end{array}$ & $\begin{array}{l}\text { Bisexual and female } \\
4\end{array}$ & & \\
\hline Rhi & Rhizome & $\begin{array}{l}\text { Stoloniferous } \\
1,2,4\end{array}$ & $\begin{array}{l}\text { Nonstoloniferous } \\
3,5,6,7\end{array}$ & & & \\
\hline Sht & Shoot & $\begin{array}{l}\text { Dimorphic } \\
1,2,4\end{array}$ & $\begin{array}{l}\text { Monomorphic } \\
3,5,6,7\end{array}$ & & & \\
\hline Lvs & Leaves & $\begin{array}{l}\text { Dimorphic } \\
3,5,6,7\end{array}$ & $\begin{array}{l}\text { Monomorphic } \\
1,2,4\end{array}$ & & & \\
\hline Flr & Flower & $\begin{array}{l}\text { Bisexual } \\
1,5,6,7 \\
\end{array}$ & $\begin{array}{l}\text { Unisexual } \\
2,3\end{array}$ & $\begin{array}{l}\text { Bisexual and unisexual } \\
4\end{array}$ & & \\
\hline ClxL & Calyx lobe & $\begin{array}{l}\text { oblong-acute } \\
1,5\end{array}$ & $\begin{array}{l}\text { Oblong-obtuse } \\
2,3\end{array}$ & $\begin{array}{l}\text { Linear- lanceolate } \\
4,6\end{array}$ & $\begin{array}{l}\text { Elliptic- } \\
\text { lanceolate } \\
7 \\
\end{array}$ & \\
\hline Co & Corolla & $\begin{array}{l}\text { White with yellow } \\
\text { throat } \\
1,4,5,6,7\end{array}$ & $\begin{array}{l}\text { white } \\
2,3\end{array}$ & & & \\
\hline PetL & Petal lobe & $\begin{array}{l}\text { Fimbriately toothed } \\
1,2,3,5,6,7\end{array}$ & $\begin{array}{l}\text { Undulate } \\
4\end{array}$ & & & \\
\hline PetLS & $\begin{array}{l}\text { Shape of petal } \\
\text { lobe }\end{array}$ & $\begin{array}{l}\text { Obtuse or retuse } \\
4\end{array}$ & $\begin{array}{l}\text { ovate to lanceolate } \\
1,6,7\end{array}$ & $\begin{array}{l}\text { Oblong or obtuse } \\
3\end{array}$ & $\begin{array}{l}\text { oblong or elliptic } \\
2,5\end{array}$ & \\
\hline HrPL & $\begin{array}{l}\text { Presence of } \\
\text { hairs on petal } \\
\text { lobe }\end{array}$ & $\begin{array}{l}\text { Present } \\
1,2,5,6,7\end{array}$ & $\begin{array}{l}\text { Absent } \\
3,4\end{array}$ & & & \\
\hline Stm & Stamen & $\begin{array}{l}\text { Dimorphic } \\
1\end{array}$ & $\begin{array}{l}\text { Monomorphic } \\
2,3,4,5,6,7\end{array}$ & & & \\
\hline ClrA & $\begin{array}{l}\text { Colour of } \\
\text { Anther }\end{array}$ & $\begin{array}{l}\text { Yellow } \\
4,5\end{array}$ & $\begin{array}{l}\text { Pale purple } \\
1,2\end{array}$ & $\begin{array}{l}\text { Blue } \\
3\end{array}$ & $\begin{array}{l}\text { Black with } \\
\text { yellow } \\
6\end{array}$ & $\begin{array}{l}\text { Cream } \\
7\end{array}$ \\
\hline NoSt & No. of stamens & $\begin{array}{l}\text { Three or four } \\
5,7\end{array}$ & $\begin{array}{l}\text { Four } \\
6\end{array}$ & $\begin{array}{l}\text { Five } \\
2,3,4 \\
\end{array}$ & $\begin{array}{l}\text { Four to eight } \\
1\end{array}$ & \\
\hline ISG & $\begin{array}{l}\text { Inter staminal } \\
\text { gland }\end{array}$ & $\begin{array}{l}\text { Present } \\
1,2,3,4,5,6\end{array}$ & $\begin{array}{l}\text { Absent } \\
7\end{array}$ & & & \\
\hline $\mathrm{ArC}$ & Stigmatic hair & $\begin{array}{l}\text { Prsent } \\
2,3\end{array}$ & $\begin{array}{l}\text { Absent } \\
1,4,5,6,7\end{array}$ & & & \\
\hline Stl & Style & $\begin{array}{l}\text { Heterostylous } \\
1\end{array}$ & $\begin{array}{l}\text { Homostylous } \\
2,3,4,5,6,7\end{array}$ & & & \\
\hline $\mathrm{HgG}$ & $\begin{array}{l}\text { Hypogynous } \\
\text { gland }\end{array}$ & $\begin{array}{l}\text { Hairy } \\
1,2,4,6\end{array}$ & $\begin{array}{l}\text { Hairless } \\
3,5\end{array}$ & $\begin{array}{l}\text { Absent } \\
7\end{array}$ & & \\
\hline Fr & Fruit & $\begin{array}{l}\text { Ellipsoid } \\
1,5\end{array}$ & $\begin{array}{l}\text { Sub globose } \\
2\end{array}$ & $\begin{array}{l}\text { Obovoid } \\
3\end{array}$ & $\begin{array}{l}\text { Oblong } \\
4,7\end{array}$ & $\begin{array}{l}\text { Oblong to } \\
\text { obovoid } \\
6\end{array}$ \\
\hline $\mathrm{SeO}$ & $\begin{array}{l}\text { Seed } \\
\text { ornamentation }\end{array}$ & $\begin{array}{l}\text { Tuberculate } \\
2,3,4,5,6\end{array}$ & $\begin{array}{l}\text { Smooth } \\
1,7\end{array}$ & & & \\
\hline SeS & Seed shape & $\begin{array}{l}\text { Discoid } \\
1,4,5,6,7\end{array}$ & $\begin{array}{l}\text { Obovate or ellipsoid } \\
2\end{array}$ & $\begin{array}{l}\text { Obovoid } \\
3\end{array}$ & & \\
\hline PoS & Pollen shape & $\begin{array}{l}\text { Prolate-spheroidal } \\
1,3,4,6\end{array}$ & $\begin{array}{l}\text { Prolate-spheroidal to } \\
\text { subprolate } \\
2\end{array}$ & $\begin{array}{l}\text { Oblate-spheroidal } \\
5,7\end{array}$ & & \\
\hline
\end{tabular}


Table 4. Character states of the OTUs

\begin{tabular}{cccccccc}
\hline \multirow{2}{*}{ Character codes } & \multicolumn{7}{c}{ Codes for OTUs } \\
\cline { 2 - 8 } & $\mathbf{1}$ & $\mathbf{2}$ & $\mathbf{3}$ & $\mathbf{4}$ & $\mathbf{5}$ & $\mathbf{6}$ & $\mathbf{7}$ \\
\hline Hab & 3 & 1 & 2 & 1 & 2 & 2 & 2 \\
\hline Plt & 1 & 2 & 2 & 3 & 1 & 1 & 1 \\
\hline Rhi & 1 & 1 & 2 & 1 & 2 & 2 & 2 \\
\hline Sht & 1 & 1 & 2 & 1 & 2 & 2 & 2 \\
\hline LvS & 2 & 2 & 1 & 2 & 1 & 1 & 1 \\
\hline Flr & 1 & 2 & 2 & 4 & 1 & 1 & 1 \\
\hline ClxL & 1 & 2 & 2 & 3 & 1 & 3 & 4 \\
\hline Co & 1 & 2 & 2 & 1 & 1 & 1 & 1 \\
\hline PetL & 1 & 1 & 1 & 2 & 1 & 1 & 1 \\
\hline PetLS & 2 & 4 & 3 & 1 & 4 & 2 & 2 \\
\hline HrPL & 1 & 1 & 2 & 2 & 1 & 1 & 1 \\
\hline Stm & 1 & 2 & 2 & 2 & 2 & 2 & 2 \\
\hline ClrA & 2 & 2 & 3 & 1 & 1 & 4 & 5 \\
\hline NoSt & 4 & 3 & 3 & 3 & 1 & 2 & 1 \\
\hline ISG & 1 & 1 & 1 & 1 & 1 & 1 & 2 \\
\hline ArC & 2 & 1 & 1 & 2 & 2 & 2 & 2 \\
\hline Stl & 1 & 2 & 2 & 2 & 2 & 2 & 2 \\
\hline HgG & 1 & 1 & 2 & 1 & 2 & 1 & 3 \\
\hline Fr & 1 & 2 & 3 & 4 & 1 & 5 & 4 \\
\hline Se & 2 & 1 & 1 & 1 & 1 & 1 & 2 \\
\hline SeS & 1 & 2 & 3 & 1 & 1 & 1 & 1 \\
\hline PoS & 1 & 2 & 1 & 1 & 3 & 1 & 3 \\
\hline & & & & & &
\end{tabular}

the basis of morphological data (including pollen, seed and flavonoid data) and molecular data (10) the floating-leaved genus Nymphoides was reported as monophyletic, except for $N$. exigua. Based on the morphological and molecular study the Indian species of Nymphoides viz. $N$. hydophylla, $N$. parvifolia and $N$. sivarajanii were closely related to $N$. krishnakesara and $N$. macosperma (12). The phenetic analysis of seven species of Nymphoides spp. gives a picture on the affinities of Nymphoides spp. in Kerala. The results shows that $N$. parvifolia and $N$.

Table 5. Percent disagreement between OTU's under study

\begin{tabular}{cccccccc}
\hline \multirow{2}{*}{ OTU } & \multicolumn{7}{c}{ OTU's arranged as per their numerical codes } \\
\cline { 2 - 8 } & $\mathbf{1}$ & $\mathbf{2}$ & $\mathbf{3}$ & $\mathbf{4}$ & $\mathbf{5}$ & $\mathbf{6}$ & $\mathbf{7}$ \\
\hline $\mathbf{1}$ & 0.00 & 0.64 & 0.86 & 0.59 & 0.55 & 0.50 & 0.59 \\
\hline $\mathbf{2}$ & 0.64 & 0.00 & 0.50 & 0.55 & 0.68 & 0.68 & 0.82 \\
\hline $\mathbf{3}$ & 0.86 & 0.50 & 0.00 & 0.68 & 0.55 & 0.55 & 0.68 \\
\hline $\mathbf{4}$ & 0.59 & 0.55 & 0.68 & 0.00 & 0.64 & 0.55 & 0.73 \\
\hline $\mathbf{5}$ & 0.55 & 0.68 & 0.55 & 0.64 & 0.00 & 0.32 & 0.32 \\
\hline $\mathbf{6}$ & 0.50 & 0.68 & 0.55 & 0.55 & 0.32 & 0.00 & 0.36 \\
\hline $\mathbf{7}$ & 0.59 & 0.82 & 0.68 & 0.73 & 0.32 & 0.36 & 0.00 \\
\hline
\end{tabular}

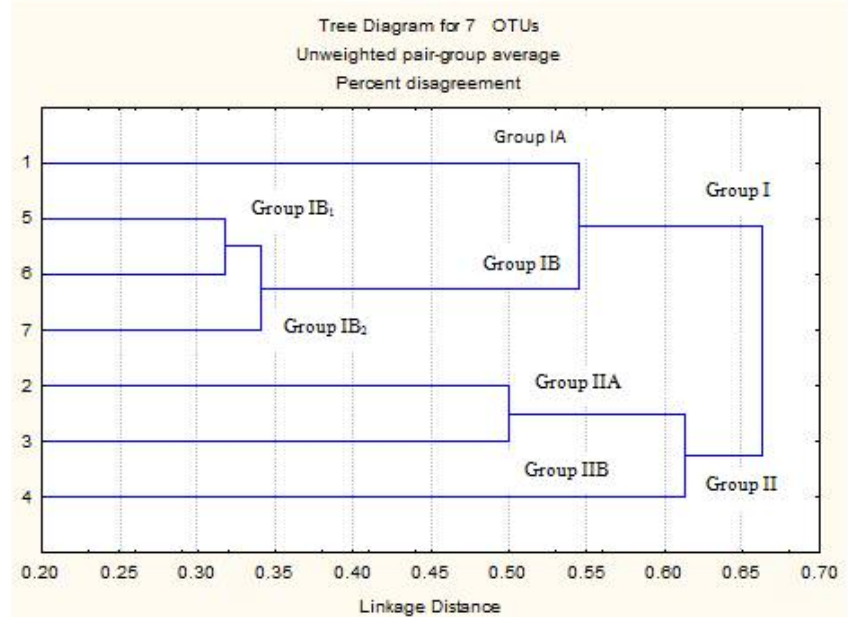

Fig 3. Dendrogram of seven species of Nymphoides in Kerala 1. N. indica, 2. N. macrosperma, 3. N. krishnakesara, 4. N. hydrophylla, 5. N. parvifolia, 6. N. balakrishnanii, 7. N. palyii. balakrishnanii are closely related species and shows highest affinities. N. krishnakesara and $N$. macrosperma in Group II also shows high affinity. Nymphoides spp. of Kerala shows two groups showing 66\% dissimilarities.

\section{Specimens examined}

a. Nymphoides indica: INDIA. Kerala: Kottayam District, Vaikom, 21 April 1988, Swaminathan M. S. 88274 (CAL!). Trichur District, Paliappara, Chalakkudy, 22 Septmeber 1982, Ramamurthy 74703; ibid., Ramamurthy 74863 (CAL!). Alappuzha District, Kanichukulangara junction, s.die, Sunil C. N. 2210 (CALI!); ibid., Angadikal, s.die, Sunil C. N. 2624 (CALI!); Kasaragod District, Peelikode, 16 February 2007, Suresh K. K 03152 (MBGH!). Kozhikode District, Olavanna, 25 September 1999, Krishnan P. N. 825 (MBGH!); ibid., 27 November 2015, Pavisha P. 12370 (MBGH!); Oorkadavu, 27 June 2008, Suresh K. K. 03994 (MBGH!); Pantheerankavu, 21 December 2016, Pavisha P. 12375 (MBGH!). Kasaragod District, Peelikode, 16 February 2007, Suresh K. K. 03317 (MBGH!); Kayyur, 05 February 2007, Suresh K. K. \& Jaris P. K. 04405 (MBGH!); ibid., s. die, Suresh K. K. \& Jaris P. K. 03181 (MBGH!).

b. Nymphoides macrosperma: INDIA. Kerala: Kozhikode District, Olavanna, 14 July 2006, Krishnan P. N. 2064 (MBGH!); Pantheerankavu, 22 February 2016, Pavisha P. 12372 (MBGH!).

c. Nymphoides krishnakesara: INDIA. Kerala: Kannur District, Madaippara, 25 October 1988, Joseph K. T. 43001 (MH!); ibid., 20 September 2005, Krishnan P. N. \& Ansari R. 2563 (MBGH!); ibid., 04 September 2008, Suresh K. K. 4004 (MBGH!); ibid., 12 December 2016, Pavisha P, Rajilesh V. K. \& Ajesh P. P. 12373 (MBGH!); Payyannur, 29 July 2009, Suresh K. K. 4964 (MBGH!); ibid., 07 October 2008, Suresh K. K. 4288 (MBGH!); ibid., 04 September 2008, Suresh K. K. \& Jaris P. K. 3317 (MBGH!); Korom, 15 January 2019, Pavisha P, Jaseela V. T \& Sinisha P. 17748 (MBGH!).

d. Nymphoides hydrophylla: British Burma, 04 January 1971, S Kurz. 2264 (CAL - Holotype!). INDIA. Kerala: Palakkad District, Elavanchery, 02 November 1976, Vajravelu M. S. 48836 (CAL!). Kannur District, Ezhuvamalai, 17 December 1979, Ramachandran 65266 (CAL!); ibid., 20 July 1981, Ansari R. 70966 (CAL!). Thrissur District, Chalakudi, 15 September 1976, Ramamurthy 48535 (CAL!) Thiruvananthapuram District, Pulimath, 02 December 1977, Mohanan 52675 (CAL!). Kasaragod District, Peelikode, 06 February 2007, Suresh K. K. \& Jaris P. K. 03162 (MBGH!); Kayyur, 06 February 2007, Suresh K. K. \& Jaris P. K. 03188 (MBGH!). Kollam District, 16 February 2007, Suresh K. K. 03229 (MBGH!). Idukki District, Moolamattam, 17 December 2008, Suresh K. K. 04436 (MBGH!). Malappuram District, Koottumoochi, 18 November 2011, Anoop K. P. \& Hareesh K. T. 6537 (MBGH!); Kozhikode District, Palazhi, 22 February 2016, Pavisha P. 12371 (MBGH!).

e. Nymphoides parvifolia: INDIA. Kerala: Kasaragod, Beemanadi, 27 September 1982, Ansari $R$. 74328 (CAL!); ibid., 18 December 2008, Suresh K. K. 4954 (MBGH!); Kayyur, 07 December 2018, Pavisha $P$, Hridhya P \& Ajesh P. P. 15813 (MBGH!). 
f. Nymphoides balakrishnanii: INDIA. Kerala: Kasaragod, Koovappara, 24 January 2019, Pavisha P. 17747 (MBGH!).

g. Nymphoides palyii: INDIA. Kerala: Kasaragod, Perla, 19 September 2016, Anoop K. P. \& Rajilesh V. K. 14595 (MBGH!); ibid., 07 December 2018, Pavisha P, Hridhya P. \& Ajesh P. P. 15831 (MBGH!).

\section{Authors' contributions}

PP carried out the field studies, identified the specimens and prepared the manuscript. MPV and PNS confirmed the species identity and helped in the manuscript preparation. All authors read and approved the final manuscript.

\section{Acknowledgements}

The authors are thankful to the Kerala State Council for Science Technology \& Environment (KSCSTE) for financial support. The authors are thankful to the Director, KSCSTE Malabar Botanical Garden \& Institute for Plant Science for providing the facilities. We also acknowledge the support and help provided by Dr. Mohanan K. V., Professor of Genetics, Calicut University in the preparation of the Dendrogram.

\section{Competing Interest}

The authors declare that they have no competing interests.

\section{Supplementary file \\ Supplementary Table 1}

\section{References}

1. Aston HI. Seed morphology of Australian species of Nymphoides (Menyanthaceae). Muelleria. 2003;18:33-65. https://doi.org/10.1097/00152193-200306000-00050

2. Biju P, Josekutty JE, Haneef AR, Augustine J. A new species of Nymphoides Séguier (Menyanthaceae) from the lateritic plateau of South India. Taiwania. 2016;61(3):218-20

3. Biju P, Josekutty JE, Haneef AR, Augustine J. Nymphoides krishnakesara var. bispinosa (Menyanthaceae), a new taxon from the lateritic plateau of Northern Kerala, India. Telopea. 2017;20:5-10

4. Hill T, Lewicki P. Statistics methods and applications. Statsoft Inc USA; 2006

5. Ornduff R. Neotropical Nymphoides (Menyanthaceae): MesoAmerican and West Indian species. Brittonia. 1969;21:346-52. https://doi.org/10.2307/2805761

6. Pham-Hoang H. Cyyco Vietnam (An illustrated flora of Vietnam). Vol. 2. Santa Ana, California: Mekong Printing; 1993

7. Raynal A. Le genre Nymphoides (Menyanthaceae) en Afrique et aMadagascar. 2e partie: Taxonomie. Adansonia. 1974;14:40558. https://doi.org/10.12681/makedonika.593

8. Sivarajan VV, Joseph KT. The genus Nymphoides Séguier (Menyanthaceae) in India. Aquat Bot. 1993;45:145-70. https://doi.org/10.1016/0304-3770(93)90019-S

9. Sokal RR, Michener CD. A statistical method for evaluating systematic relationships. Univ. Kans. Sci. Bull.1958;38:1409-38

10. Tippery NP Les, DH Padgett DJ, Jacobbs SW. Generic circumscription in Menyanthceae: A phylogenetic analysis. $\begin{array}{lll}\text { Systematic } & \text { Botany. 2008;33(3):598-612. }\end{array}$ https://doi.org/10.1600/036364408785679851

11. Tippery NP, Les DH. A new genus and new combinations in Australian Villarsia (Menyanthaceae). Novon. 2009;19:40411. https://doi.org/10.3417/2007181

12. Tippery NP, Les DH. Phylogenetic Relationships and Morphological Evolution in Nymphoides (Menyanthaceae). $\begin{array}{lll}\text { Systematic } & \text { Botany. }\end{array}$ https://doi.org/10.1600/036364411X605092 